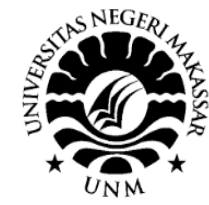

\title{
Early Stimulation in Female Heads Of Household
}

\author{
Cruz García Lirios \\ Universidad Autónoma del Estado de México. Coyoacan, Distrito Federal, MX \\ Email: bundestrans@icloud.com
}

\begin{abstract}
The exploration of the structure of relations between variables that explain the process of early stimulation was the objective of the present work. A cross-sectional quantitative study was carried out with a non-probabilistic selection of 100 students, considering their participation in a family planning and entrepreneurship program. A composition of eight factors was found: values, beliefs, perceptions, knowledge, abilities, attitudes, intentions and behaviors that explained $45 \%$ of the total variance, but the design of the research limited the findings to the scenario suggesting the inclusion of factors that Literature identifies as parenting styles in terms of volitional theories.
\end{abstract}

Keywords: volitional development, mesosystem, family, early stimulation, structure

\section{INTRODUCTION}

The purpose of this study is to establish the impact of a public health program on early stimulation as a quality of life factor in the initial education system. The psychosocial model of public health is made up of three dimensions around which social services interact with prevention and immediate attention.

The National Institute of Statistics and Geography (INEGI), in its report for the year 2010 warns a rising trend of births if the 2005 data is considered, compared to the data for the year of 2008. The trend of births would also be related to the incidence of deaths which has also been increasing, considering the 1985 data compared to the data for the year corresponding to 2004. The case of children under three years is striking, since diabetes mellitus is the main cause of death, followed by ischemic heart and cardiovascular diseases. In this sense, the prevention and attention of diseases from the early stimulation seem to be relevant; especially if it is considered that an exercise session could help to activate the propensity to take care of health in families (Campos, Cordero, Rojas and Vargas, 2005).

Early stimulation refers to a sequential, continuous, systematic and regulated treatment to improve sensory abilities, without compromising the development of the nervous and cognitive system in the first years of a child's life. It is important to note that the state of the art related to early stimulation focuses on different dimensions: emotional, cognitive, behavioral, moral and social. In this sense, the studies on early stimulation, despite their theories and interdisciplinary methods, exhibit an eminently psychological approach (Castellanos, García and Mendieta, 1999). 
In effect, the Theory of Moral Development (TDM), one of the theories on which early stimulation is based on three different stages: 1) preconventional, 2) conventional and 3) postconventional. It is a cultural-cognitive structure in which moral principles interact within a reference group or membership (Cere, Trenado and Pons, 2006).

The conflict of endo-group versus exo-group principles generates changes around the socialization and identity of individuals. That is, moral principles not only guide behavior but also define emotional, cognitive and behavioral changes based on judgments of dilemmas consisting of everyday situations. Moral principles are the result of interaction between groups and their members. In the case of families, parents educate their children by encouraging ethical and moral values or principles, although in appearance, only auditory, visual, kinestic, emotional or sensory stimulation is performed (Escalona and Lopez, 2011).

In this sense, TDM is an extension of the Theory of Development Statutes (TED) around the moral judgment of children that is cognitive and social. The cognitive moral development as the last stage is the first stage of evolutionary social development child (Garcia, 2011).

Even, the TDM incorporates the levels of interaction proposed by the Theory of the Ecology of Human Development; microsystem, mesosystem, exosystem and macrosystem in which groups and individuals interact with situational environments. In effect, the TDM poses an evolutionary growth from the interaction with the environment of the group to which the children belong. This environment is presented in situations that must be judged. As dilemmas of choice, the situations are directly related to evaluations and negative or positive evaluations of the behavior of similar people in homogeneous situations (Garcia, Montero, Bustos, Carreon and Hernandez, 2012).

The early stimulation has its origin in Jean Piaget's Theory of Development Stages, since it has been proposed as a systematic process of parental influence on the abilities, abilities and competences of its children. However, TED only explained cognitive behavioral evolution. In this sense, moral development explains the cultural socio-cognitive development and Theory of Moral Development (MDD) raisessistem to $\mathrm{s}$ interaction between the groups to which children belong and environmental and contextual situations they are exposed choosing, judging and evaluating said conventions (Garcia, 2012).

Psychological studies of early stimulation have established stages, phases, areas, factors and dimensions of intervention to demonstrate the direct, positive and significant effect of strategies and techniques of parental stimulation-tutorials on the evolutionary development of children and adolescents. Unearly stimulation program focused on family about the importance of family dynamics include school performance as an effect of the care of the physical, emotional, cognitive and motor health. The program was evaluated by judges who gave it content validity (García, Tacoronte, Sarduy, Abdo, Galvizu, Torres and Leal, 2000).

In an association between the detected needs of the child and his chronological age of sensorimotor development, the intervention of the tutor-therapist was decisive. Given that the diagnosis of children's needs corresponds with the chronological age of sensory and motor development, the role of the specialist is fundamental in influencing child development. The decrease in cerebral palsy from early stimulation. During the treatment, three areas of intellectual development of great importance intervened: audiovisual, physical rehabilitation and congenital defectology. At the end of the intervention, they found an increase in the coefficient of cognitive behavioral development (González, 2007). 
The risk factors around the birth of an experimental group before and after the stimulation warn a decrease in the risk factors of the experimental group compared to the control group. During the 40-week early stimulation period, the experimental group achieved a higher percentage of cognitive-behavioral development in reference to the control group (Jara and Roda, 2010).

Three stages of the study: diagnosis, planning and intervention describe in the effect of the ludoterapia in children on the part of personnel of infirmary and paediatrics of the General Hospital of San Rafael de Alajuela, Costa Rica. In the first stage, they demonstrated that health professionals have basic knowledge that, due to their degree of specialization, has affected the anxiety levels of hospitalized children and their families (Manzano, Per era, Ortiz and Arena, 1990).

These are some of children's behavior histories; 1 . positive and neutral, 2. sensitive and attentive; this in direct relation with four consequent maternal conducts: sensitive attentive, sensitive affectionate, intrusive affectionate and instruction to observe the differences that were significant between two groups; low in coordination and high in coupling. In almost all combinations significant differences were demonstrated, only in the high-coupling group the intrusive response was spurious in relation to the antecedent infantile behavior (Maris cal, Nieva and López, 2010).

In contrast, five areas of early stimulation: mathematical, social, personal, cognitive and motor; they defined a structured educational system aimed at the development of children. Subsequently, he demonstrated five behavioral effects of early stimulation: socialization, identity, responsibility, expressiveness and responsibility. However, some behaviors of self-control and hygiene could not be established. In this sense, early stimulation was related to divergent thinking, in clear contrast to logical-mathematical thinking. It should be noted that early stimulation encouraged reading and writing, despite the limitations of the tutors-therapists in terms of knowledge of updating programs and intervention strategies (Martinez, Garcia, Poblano and Madlen, 2010).

There are areas, dimensions or factors of early stimulation: visual, auditory, tactile, olfactory, vestibular and sensory; correspond with special abilities of children to design a space stimulation. The design contemplated the analogy of a recreational space in which materials would be the main stimulators of child development. The proposal also includes the interrelation between spaces taking into account the creativity of children who could interact with other children with special abilities (Molina and Banguero, 2008).

The association is significant in four areas of stimulation: motor, cognitive, readingwriting and social. The first dimension refers to the stimulation and its systematic kinesthetic effects in which the child acquires skills and, above all, competences or effective responses to specific problems. The second factor refers to the development of basic psychological processes such as language, memory and perception. In the third construct, it alludes to the intelligence or intellectuality coefficient, which were evidence of the comprehension abilities of texts. Finally, in the last area, stimulation is a determinant of social, communicative, discursive and interpretive skills (Moreno, 2009).

The association is significant between the five phases of early stimulation. In the first diagnostic phase, interviews with parents about the abilities of their children. In the second, a curricular structure in which early stimulation would be included to influence the child's integral development. In the third phase, the adaptive skills whose objectives were learning to know, do, live and be. In the fourth phase, mediation strategies in which the early stimulation of the institution would be complemented with family intervention strategies. In the fifth phase, socialization strategies and family identification (Pando, Aranda, Amezcua, Salazar and Torres, 2004). 
There are direct and significant associations between parental self-reports and the speech observations of their children. types of speech: irregular morphology and regular morphology, which correlated positively with the word emission intervals and grammatical development protocols. Both morphologies increased to the extent that speech lengths increased. That is, the development of language is associated with the stimulation of parents towards their children (Tejada and Otalora, 2006).

Stages of early stimulation to demonstrate the significant differences between techniques corresponding to each stimulation. In the first stage from zero to three months, the stimulation was more auditory than visual in which the gesticulations are important, but more so are the talks, massages, songs, games and lullabies. In the second stage of three to six months, stimulation through care, company, calm and conversation were essential for the acquisition of language through babbling. In the third stage from six to nine months, auditory stimulation tends to be displaced by kinesthetic stimulation because the torso and parietal exercises developed their motor skills. In the fourth stage from nine to twelve months, the stimulation is still auditory and visual, but to a greater extent it is kinesthetic since the babbling, the auditory and visual training, as well as the games are essential for the development. In the fifth stage from 12 to 18 months the language begins to develop with the reinforcement of concepts in which words and numbers have a significant effect on the learning of everyday activities through imitation. In the sixth stage from 18 to 24 months, the stimulation consisted in encouraging the narration with three-phase words. In the seventh stage from 24 to 30 months, verbal auditory stimulation is carried out with the reading of books, children's games and mnemonic exercises. Finally, in the eighth stage from 30 to36 months, the stimulation was carried out with the help of films and telephones in which the messages or auditory, visual and verbal contents were exercised (Garcia, 2011).

A significant association between early stimulation and psychomotor development over four quarters of intervention. At first, 83 percent of the mothers had insufficient knowledge of the stimulation process. At the end of the intervention only four percent had the same level of knowledge (Torres, Montilva, Ramírez, Zerpa, Zapata, García, Papale, Puertas, Franco and Palencia, 2010).

The psychological studies of early stimulation have shown the causal associative relationships between the social and institutional value judgments about the value judgments of families through the unfavorable or favorable attitudes of parents regarding cognitive, behavioral and social development of their children. In as much, valorative judgments, the attitudes towards the processes of early stimulation are evidence of the sociocultural environmental impact on the emotions, cognitions and family and parental behaviors. In other words, the development of children depends on the situations and contexts that surround their families. This influence is mediated by the attitudes, decisions, intentions and instructive educational strategies of their parents (Garcia, 2012).

\section{METHOD}

A cross-sectional quantitative study was carried out. A non-probabilistic selection was made among 258 students who were studying, on a regular basis, in the Huehuetoca Professional Academic Unit of the Autonomous University of the State of Mexico. The piloting applied to students for practical purposes, to any group or conglomerate, more or less homogeneous and that performs in a peaceful and constructive manner.

Now, the central purpose of turning to students who study some of the various degrees taught in that Academic Unit, belonging to the Autonomous University of the State of Mexico, was simply to pilot an instrument alluding to early stimulation. This with 
the purpose of getting to homogenize a later instrument. That is, to make the instrument appropriate to the culture, idiosyncrasies, values and customs of the municipality of Xilitla, SLP, with single mothers who are entrepreneurs and dedicated to coffee growing. Of course, the fulfillment of the previously indicated requirements always remained present. It is convenient to point out that once obtained the results of the piloting, later, the different interviews could be carried out, with the single mothers dedicated to coffee growing in the municipality of Xilitla, SLP, in an optimal way and with the least possible setbacks.

We used the short version of the Adoption Scale for the Treatment of Chronic Degenerative Diseases of Olguín-González (2013) which includes 72 statements about norms $($ alpha $=0,724)$, values $($ alpha $=0,789)$, beliefs $($ alpha $=0,761))$, virtues $($ alpha $=$ $0.751)$, spaces $($ alpha $=0.790)$, quality $($ alpha $=0.841)$, perceptions $($ alpha $=0.829)$, knowledge $($ alpha $=0.895)$, skills $($ alpha $=0.886)$, attitudes $($ alpha $=0.856)$, decisions $($ alpha $=0.719)$, intentions (alpha 0.725) and behaviors (alpha 0.826) with five response options ranging from "it does not look like my situation" to "it is very similar to my situation".

The participants were interviewed and surveyed in the facilities of the Municipal Library "Florencio Salazar Martínez". They were informed that the results of the investigation would not affect their school situation positively or negatively. They were asked to honestly and honestly answer the questions and assertions. Likewise, they were asked to participate more students from their own campus, for this purpose the attendance to the library in question was made more expensive. The truth is that the answer - perhaps because of the novelty of the case - was quite successful. Finally, they were invited to consult the results in the final report of the research group. The data were processed in the Statistical Package for Social Sciences (SPSS for its acronym in English) and the Software of Analysis of Structural Moments (AMOS for its acronym in English) in its versions 10 and 6.0

The parameters of kurtosis, Cronbach's alpha, KMO coefficients, Bartlett's test, factorial weights, Pearson correlations, "phi" covariances, "beta" and "range" weights, as well as adjustment and residual indexes were used in order to contrast the model of relationships specified with the observed data.

Normal. The value of the kurtosis close to the unit was assumed as evidence of the normal distribution of respondent responses with respect to the assertions, which measure the study variables in an instrument with response options and interval measurement levels.

Validity. The KMO coefficients higher than 0,600 and the Bartlett test with significance levels less than 0.050 were assumed as evidence of product moment correlations that facilitated the exploratory factor analysis of principal axes with promax rotation. Subsequently, factor weights greater than 0,300 were considered as evidence of the maximization of variance in terms of the factors derived from the exploratory analysis. The explained variance percentages higher than 0.20 were assumed as evidence of acceptance of the null hypothesis.

Reliability. The Cronbach's alpha value greater than 0.60 was assumed to be sufficient to demonstrate the internal consistency of the indicators with respect to the general scale and the particular subscales. The correlation product of the moment: higher than 0.90 was considered as an evidence of collinearity and multicollinearity, which means that the items are similar in terms of their contents.

Correlation. Pearson's r values close to unity and zero were discarded from further analysis since they signify collinear or spurious relationships. On the other hand, those 
values higher than 0.30 and lower than 0.90 were assumed as evidence of dependency relationships.

Covariance The "phi" values between 0.30 and 0.90 were identified as evidence of dependency relationships for the case of categorical variables or in combination with continuous variables.

Structure. The "beta" values between exogenous and endogenous variables between 0.30 and 0.90 were assumed as evidence of dependency relationships. Similarly, "gamma" values between endogenous variables close to zero or unity were discarded from further analysis.

Adjustment. The Adjustment Goodness Index (GFI) close to the unit was assumed as evidence of adjustment and acceptance of the null hypothesis. On the other hand, values lower than 0.975 were considered as evidence of rejection of the null hypothesis and acceptance of the alternative hypothesis.

Residual. The values close to zero were assumed as evidence of adjustment between the specified relationships and the data obtained, therefore, the null hypothesis of adjustment between both models was accepted. In contrast, values higher than 0.007 were considered as evidence of rejection of the null hypothesis.

\section{RESULTS}

Table 1 shows the parameter values that demonstrate the normal distribution, reliability and validity of the instrument that measured the early stimulation.

Table 1. Descriptive the instrument

\begin{tabular}{|c|c|c|c|c|c|c|c|c|c|c|c|c|}
\hline $\boldsymbol{R}$ & $M$ & $S$ & $C$ & $\mathbf{A}$ & $F 1$ & $F 2$ & $F 3$ & F4 & $F 5$ & F6 & F7 & $F 8$ \\
\hline$r 1$ & 4.3 & 1,3 & 1,3 & ,76 & ,35 & & & & & & & \\
\hline$r 2$ & 4.5 & 1,2 & 1,2 & ,77 & ,46 & & & & & & & \\
\hline$r 3$ & 4.8 & 1.0 & 1.4 & ,78 &, 56 & & & & & & & \\
\hline$r 4$ & 4.0 & 1.1 & 1,5 & ,79 & ,60 & & & & & & & \\
\hline$r 5$ & 4.4 & 1,2 & 1.7 & ,70 & & ,67 & & & & & & \\
\hline$r 6$ & 4.6 & 1,3 & 1.9 &, 72 & & ,51 & & & & & & \\
\hline$r 7$ & 4.4 & 1,5 & 1.6 &, 73 & & ,42 & & & & & & \\
\hline$r 8$ & 4.3 & 1.4 & 1.0 & ,75 & & ,43 & & & & & & \\
\hline r9 & 4.5 & 1,8 & 1,3 & ,76 & & & ,57 & & & & & \\
\hline r10 & 4.4 & 1,5 & 1,5 & ,78 & & & ,65 & & & & & \\
\hline$r 11$ & 4.2 & 1,8 & 1.4 &, 75 & & & ,69 & & & & & \\
\hline$r 12$ & 4.1 & 1.9 & 1.7 & ,71 & & & ,62 & & & & & \\
\hline$r 13$ & 4.0 & 1.4 & 1,5 & ,70 & & & & ,47 & & & & \\
\hline$r 14$ & 4.8 & 1,5 & 1.6 & ,73 & & & &, 52 & & & & \\
\hline$r 15$ & 4.7 & 1,3 & 1.9 &, 72 & & & & , 48 & & & & \\
\hline$r 16$ & 4.3 & 1,2 & 1.4 & ,74 & & & & ,54 & & & & \\
\hline$r 17$ & 4.5 & 1.6 & 1,3 & ,79 & & & & &, 56 & & & \\
\hline$r 18$ & 4.6 & 1.7 & 1,2 & ,78 & & & & &, 55 & & & \\
\hline$r 19$ & 4.8 & 1.0 & 1.1 & ,74 & & & & & ,51 & & & \\
\hline$r 20$ & 4.4 & 1,3 & 1.0 &, 75 & & & & & ,56 & & & \\
\hline$r 21$ & 4.1 & 1.6 & 1.9 &, 72 & & & & & & ,47 & & \\
\hline$r 22$ & 4.2 & 1,8 & 1,8 & ,71 & & & & & & ,43 & & \\
\hline$r 23$ & 4.3 & 1,2 & 1.7 & ,70 & & & & & & ,41 & & \\
\hline$r 24$ & 4.8 & 1,3 & 1.6 & ,73 & & & & & & ,59 & & \\
\hline$r 25$ & 4.3 & 1.0 & 1,8 & ,77 & & & & & & &, 52 & \\
\hline
\end{tabular}




\begin{tabular}{lrrrrrr}
$\boldsymbol{r 2 6}$ & 4.5 & 1,2 & 1,5 &, 70 &, 47 & \\
\hline $\boldsymbol{r 2 7}$ & 4.7 & 1.6 & 1.9 &, 73 &, 52 & \\
\hline $\boldsymbol{r 2 8}$ & 4.3 & 1.0 & 1.6 &, 74 &, 42 & \\
\hline $\boldsymbol{r 2 9}$ & 3.6 & 1.7 & 1.0 &, 72 &, 51 \\
\hline $\boldsymbol{r 3 0}$ & 3.6 & 1.9 & 1.6 &, 73 &, 62 \\
\hline $\boldsymbol{r 3 1}$ & 4.6 & 1.0 & 1,2 &, 71 &, 59 \\
\hline $\boldsymbol{r 3 2}$ & 3.7 & 1,8 & 1.0 &, 72 &, 48 \\
\hline
\end{tabular}

Source: Prepared with the study data; $R=$ Reactive, $M=$ Mean, $S=$ Standard Deviation, $K=$ Kurtosis, $A=$ Cronbach's Alpha, removing the value of the item. Adequacy $(K M O=$ 0,672), Sphericity $=\lceil\chi 2=235,13(24 \mathrm{gl}) p=0,000\rfloor$ Method: Main Axes, Rotation: Promax. $F 1=$ Values $(13 \%$ of the total variance explained), $F 2=$ Beliefs $(10 \%$ of the total variance explained $), F 3=$ Perceptions $(7 \%$ of the total variance explained $), F 4=$ knowledge (5\% of the total variance explained), $F 5=H$ abilities ( $4 \%$ of the total variance explained), $F 6=$ Attitudes (3\% of the total variance explained), $F 7=$ Intentions (2\% of the total variance explained), $F 8=$ Behaviors (1\% of the total variance explained).

The reliability of the scale exceeded the minimum required to be considered as consistent in different scenarios and samples. The validity obtained a preponderant intentional factor (IS) that explained $45 \%$ of the total variance.

The adequacy and sphericity reached the values required to carry out the exploratory factorial analysis of principal axes with promax rotation $\left\lceil\chi^{2}=235,13(24 \mathrm{gl}) \mathrm{p}=\right.$ $0.000 ; \mathrm{KMO}=0.662 \mathrm{~J}$

Table 2. Correlations and covariances between the factors

\begin{tabular}{|c|c|c|c|c|c|c|c|c|c|c|c|c|c|c|c|c|}
\hline & $F 1$ & $F 2$ & $F 3$ & $F 4$ & $F 5$ & F6 & F7 & $F 8$ & $F 1$ & $F 2$ & $F 3$ & $F 4$ & $F 5$ & F6 & $F 7$ & $F 8$ \\
\hline$F 1$ & 1.0 & $65 *$ & $58 * *$ & $52 *$ & $60 * * *$ &, $47 *$ &, $50 * *$ &, $62 *$ & 1.9 & 43 & 53 & 44 & 65 & 43 & 58 & 69 \\
\hline$F 2$ & & 1.0 &, $43 *$ & $67 *$ &, $59 *$ & $44 * *$ &, $63 *$ &, $66 * *$ & & 1,8 & 47 & 40 & ,50 & 47 & 57 & 67 \\
\hline F3 & & & 1.0 & $52 *$ &, $52 *$ &, $58 *$ &, $54 *$ &, $59 *$ & & & 1.9 & 56 & 51 & 54 &, 53 & 65 \\
\hline F4 & & & & 1.0 &, $51 *$ & $62 * * *$ &, $55 *$ &, $57 *$ & & & & 1.7 & 48 & 52 & 55 & 63 \\
\hline$F 5$ & & & & & 1.0 &, $59 *$ & $50 * * *$ &, $52 *$ & & & & & 1.6 & 49 & 67 & 62 \\
\hline F6 & & & & & & 1.0 &, $47 *$ & , $47 * *$ & & & & & & 1,8 & 62 & 60 \\
\hline F7 & & & & & & & 1.0 & $51 * * *$ & & & & & & & 1.7 & 64 \\
\hline$F 8$ & & & & & & & & 1.0 & & & & & & & & 1.9 \\
\hline
\end{tabular}

Source: Prepared with the study data; $F 1=$ Values, F2 $=$ Beliefs, F3 $=$ Perceptions, F4 = Knowleedge, F5 = Habilities , F6 $=$ Attitudes , F7 = Intentions , F8 $=$ Behaviors : ${ }^{*} p<, 01 ;{ }^{* *} p$ $<0.001 ; * * * p<0001$

Once the eight factors were established, we proceeded to estimate the associations and covariances between them in order to establish their dependency relationships and the emergence of a factor common to early stimulation (see Table 2 and Figure 1).

Figure 1. Structural equation modelling 


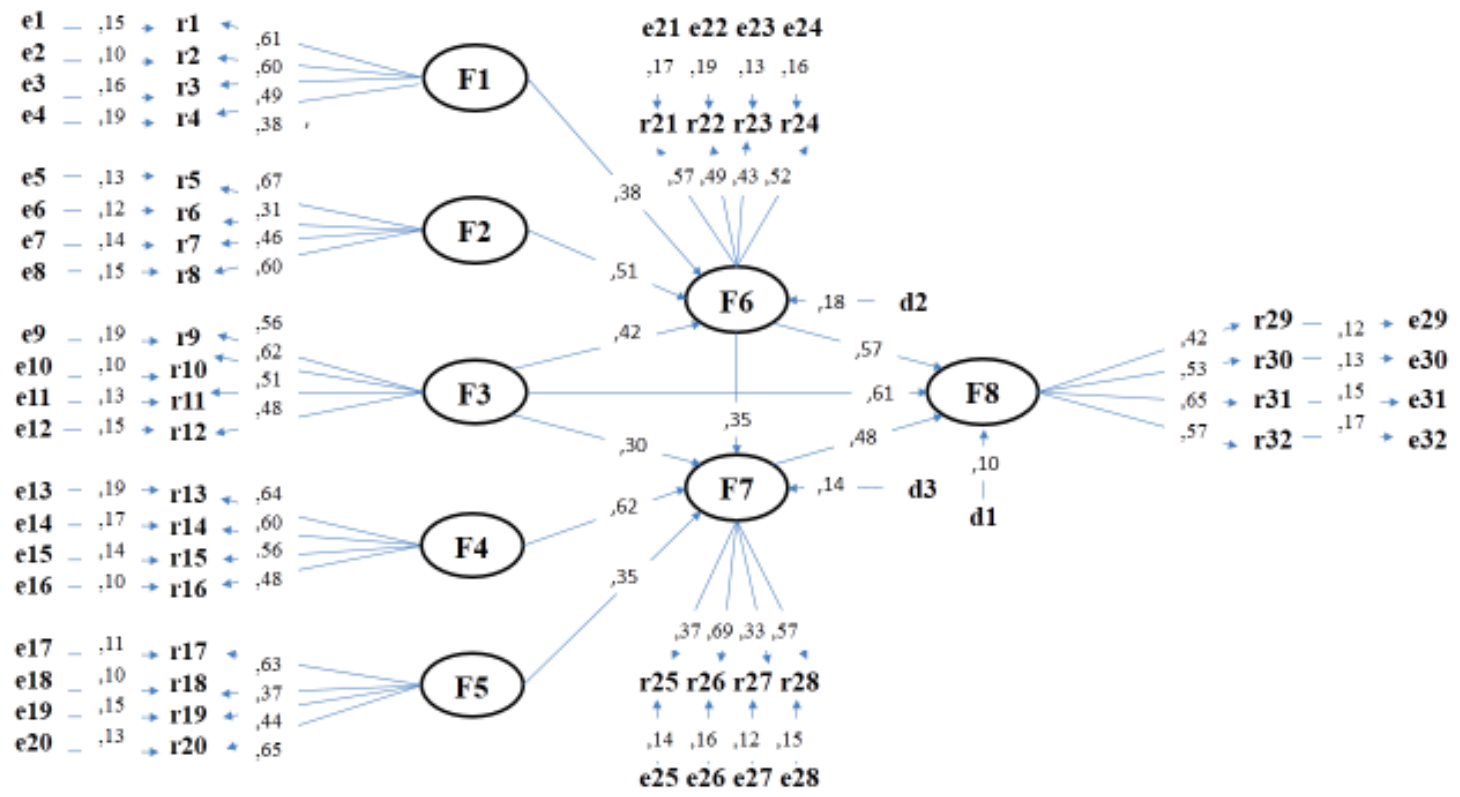

Source: Elaborated with data study; $F 1=$ Values, $F 2=$ Beliefs, $F 3=$ Perceptions, $F 4=$ Knowleedge, $F 5=$ Habilities, $F 6=$ Attitudes, $F 7=$ Intentions, $F 8=$ Behaviors: $d=$ disturbance measured factors; e 0 errors measured indicators; $\leftarrow$ relations between disturbance with factors or errors with indicators; $\rightarrow$ relations between factors and indicators

The adjustment and residual parameters $\left[\chi^{2}=14.25(23 \mathrm{gl}) \mathrm{p}=0.000 ; \mathrm{GFI}=0.975\right.$; CFI $\left.=0.970 ; \mathrm{NFI}=0.925 ; \mathrm{RMSEA}=0.004 ; \mathrm{RMR}=0.001 ; \mathrm{R}^{2}=0.256\right]$ suggest the nonrejection of the null hypothesis relative to the significant differences between the variables with respect to the model.

\section{FINAL CONSIDERATIONS}

In relation to early stimulation as a result of adopting parental parenting styles centered on permissiveness, the present study shows that the family sine is of the utmost importance, but the interrelation between parents and social workers has allowed a greater incidence in the reproduction of healthy and violence-free lifestyles, focused above all on so-called self-care, rather than family dedication or absorption.

In this sense, early stimulation as part of a treatment process is closer to adherence, rather than to a parenting style. In the present study it was highlighted that it is the norms and values that, when interacting, determine a perception of risk that encourages the adoption of early stimulation. That is, the styles of parental parenting are prone to treatments as long as their perception of risk intensifies.

In this way, the perception of risk crystallized in a norm of family prevention comes to determine an obvious more protective parenting style, based on which the adoption and implementation of early stimulation will be encouraged.

In effect, once the adoption of early stimulation is completed, it does not guarantee an implementation, but if a close group, then the parenting styles adopt the treatment will reflect that influence in their treatment or prevention protocols. This is the case of a member of the family whose illness is linked to stimulation as treatment or rehabilitation therapy.

However, the adoption of early stimulation is closer to the perception of risk than in the parenting styles, which implies a threshold of vulnerability, as in the case of first-born children with low birth weight. In the present study it has been shown that attitudes, as 
parents' evaluations regarding the health of their children, directly affects the adoption of a treatment.

It is recommended to deepen the trajectories of dependency relationships between parenting styles with emphasis on perceptions of risk and relationship with the adoption of early stimulation in future parents or prone to sexual reproduction as a community or group norm. A study of the factors that determine the perception of risk would allow an incidence in cases of unwanted pregnancy, or else, in cases in which the future parents will adopt parenting styles that will lead them to implement strategies to prevent diseases.

The contribution of the present work, embodied in this chapter, to the state of knowledge, lies in the contrast of a model for the study of early stimulation in which values turned out to be its main determinants. In reference to a correlation between selfish values and attitudes towards early stimulation, this research considers that they are the external determining values of adoption to treatment whose explanatory power is transmitted by attitudes towards the disease, carriers or health care professionals. .

However, the generality of social values, even group values such as family values, are general factors and, therefore, little explanatory of specific behaviors such as adoption to treatment. It is recommended the inclusion of other perceptual variables in which the risk would determine adoption behaviors to the treatment of a chronic-degenerative disease.

\section{REFERENCES}

Campos, C., Cordero, S., Rojas, G. and Vargas, M. (2005). Ludotherapy and early stimulation: care of the hospitalized child and the girl and her family. Nursing in Costa Rica. 12, 4-10

Castellanos, P., García, F. and Mendieta, P. (1999). Sensorimotor stimulation from the integrated model in early care. Century Zero 31, 5-13

Cerezo, M., Trenado, R. and Pons, G. (2006). Early mother-child interaction and factors that negatively affect parenthood. Psicothema $18,544-550$

Escalona, J. and López, M. (2011). Impact of an early stimulation program on low birth weight children. Pediatrics Magazine. 8, 42-72

García, C. (2011). Structure of the parental provision towards early stimulation. Journal of Psychology, 15, 1-10

García, C. (2012). Structure of sociodemographic determinants of attitude towards early stimulation. Magazine without Border, 3, 83-93

García, C., Montero, M., Bustos, M., Carreón, J. and Hernández, J. (2012). Model of the sociodemographic and cognitive determinants of early stimulation. Revista Huaricha, 9 (19), 127-147

García, M., Tacoronte, M., Sarduy, I., Abdo, A., Galvizu, R., Torres, A. and Leal, E. (2000). Influence of early stimulation in cerebral palsy. Journal of Neurology. 31, 716-719

González, C. (2007). Early stimulation programs from the teacher's perspective. Liberabit. 13, 19-27 
Jara, M. and Roda, P. (2010). Curricular proposal of adaptive skills for the early stimulation of children from 0 to 3 years of age with cognitive disabilities. Educare 1, 143-158

Manzano, M., Perera, M., Ortiz, L. and Arena, C. (1990). Design of an early stimulation program for children with hearing problems. Cuban Journal of Psychology. 7, 137143

Mariscal, S., Nieva, S. and López, S. (2010). Observe and measure early grammatical development in Spanish. Psicothema 22, 51-56

Martínez, C., García, P., Poblano, A. and Madlen, M. (2010). Early stimulation of hearing and language for children with high risk of neurological sequelae. Mexican Pediatric Record. 31, 304-310

Molina, T. and Banguero, L. (2008). Design of a sensory space for the early stimulation of children with multideficit. Journal of Biomedical Engineering. 2, 40-47

Moreno, A. (2009). Early stimulation Innovation and Educational Trends. 14, 1-9

Pando, M., Aranda, C., Amezcua, M., Salazar, J. and Torres, T. (2004). Early stimulation in children under four years of marginalized families. Mexican Journal of Pediatrics. 71, 273-277

Tejada, M. and Otalora, C. (2006). Cognitive stimulation of mothers of the popular sector. Research and Postgraduate 21, 43-68

Torres, A., Montilva, M., Ramírez, J. Zerpa, C., Zapata, E., Garcia, M., Papale, J., Puertas, M., Franco, M. and Palencia, E. (2010). Moral development in students of nursing and medicine at the Universidad Centro Occidental Alvarado. Journal of Medicine. 33, 36-41 